\title{
TNF overproduction impairs epithelial staphylococcal response in hyper IgE syndrome
}

\author{
Ian A. Myles, ${ }^{1}$ Erik D. Anderson, ${ }^{1}$ Noah J. Earland, ${ }^{1}$ Kol A. Zarember, ${ }^{2}$ Inka Sastalla, ${ }^{1}$ Kelli W. Williams, ${ }^{1}$ Portia Gough, ${ }^{1}$ \\ Ian N. Moore, ${ }^{3}$ Sundar Ganesan, ${ }^{4}$ Cedar J. Fowler, ${ }^{1}$ Arian Laurence, ${ }^{5}$ Mary Garofalo, ${ }^{2}$ Douglas B. Kuhns, ${ }^{6}$ Mark D. Kieh, ${ }^{1}$ \\ Arhum Saleem, ${ }^{1}$ Pamela A. Welch, ${ }^{1}$ Dirk A. Darnell, ${ }^{1}$ John I. Gallin, ${ }^{2}$ Alexandra F. Freeman, ${ }^{1}$ Steven M. Holland, ${ }^{1}$ and Sandip K. Datta ${ }^{1}$ \\ 'Laboratory of Clinical Immunology and Microbiology, and 'Laboratory of Host Defenses, National Institute of Allergy and Infectious Diseases (NIAID), NIH, Bethesda, Maryland, USA. ${ }^{3}$ Infectious Disease and \\ Pathogenesis Section, Comparative Medicine Branch, NIAID, NIH, Rockville, Maryland, USA. “Biological Imaging Section, Research Technology Branch, NIAID, NIH, Bethesda, Maryland, USA. ${ }^{5}$ Translational \\ Gastroenterology Unit, John Radcliffe Hospital, Oxford University, Oxford, United Kingdom. ${ }^{6}$ Applied Developmental Research Directorate, Leidos Biomedical Research Inc., Frederick National Laboratory for \\ Cancer Research, Frederick, Maryland, USA.
}

\begin{abstract}
Autosomal dominant hyper IgE syndrome (AD-HIES), or Job's syndrome, is a primary immune deficiency caused by dominantnegative mutations in STAT3. Recurrent Staphylococcus aureus skin abscesses are a defining feature of this syndrome. A widely held hypothesis that defects in peripheral Th17 differentiation confer this susceptibility has never been directly evaluated. To assess the cutaneous immune response in AD-HIES, we induced suction blisters in healthy volunteers (HVs) and patients with AD-HIES and then challenged the wound with lethally irradiated bacteria. We show that cutaneous production of IL-17A and IL-17F was normal in patients with AD-HIES. Overproduction of TNF- $\alpha$ differentiated the responses in AD-HIES from HVs. This was associated with reduced IL-10 family signaling in blister-infiltrating cells and defective epithelial cell function. Mouse models of AD-HIES recapitulated these aberrant epithelial responses to $S$. aureus and involved defective epithelial-to-mesenchymal transition (EMT) rather than a failure of bacterial killing. Defective responses in mouse models of $A D$-HIES and primary keratinocyte cultures from patients with AD-HIES could be reversed by TNF- $\alpha$ blockade and by drugs with reported modulatory effects on EMT. Our results identify these as potential therapeutic approaches in patients with ADHIES suffering $S$. aureus infections.
\end{abstract}

\section{Introduction}

Autosomal dominant hyper IgE syndrome (AD-HIES), also known as Job's syndrome, is a primary immune deficiency caused by dominant-negative loss-of-function mutations in the STAT3 gene. Recurrent Staphylococcus aureus skin abscesses (1), reminiscent of the boils that smote the biblical figure Job, are a defining feature of this syndrome. Additional features include elevated serum IgE, atopic dermatitis, recurrent pulmonary infections, bone fragility, and vascular abnormalities, all of which may be related to impaired wound healing and tissue remodeling (1-4). When a defect in the peripheral blood $\mathrm{CD} 4{ }^{+} \mathrm{T}$ cell production of IL- 17 was discovered in patients with AD-HIES (5), it was proposed that the patients' increased $S$. aureus susceptibility might be due to reduced IL-17 production. However, the hypothesis of IL-17-mediated $S$. aureus defects in patients with AD-HIES has never been directly evaluated. To assess the cutaneous immune response in AD-HIES, we induced suction blisters in healthy volunteers (HVs) and patients with AD-HIES, removed the epidermal layer, and challenged the wound with lethally irradiated bacteria (6). Here, we show that cutaneous production of IL-17A, IL-17F, and most antimicrobial peptides was normal in patients with AD-HIES, suggesting an alternative mechanism of aberrant $S$. aureus responses. We show

Conflict of interest: The authors have declared that no conflict of interest exists Submitted: April 4, 2018; Accepted: June 6, 2018.

Reference information: / Clin Invest. 2018;128(8):3595-3604.

https://doi.org/10.1172/JCl121486. that overproduction of TNF- $\alpha$ differentiated the responses of patients with AD-HIES from those of HVs and was associated with epithelial dysfunction, as indicated by markers of epithelial-tomesenchymal transition (EMT). These results are consistent with prior studies demonstrating intact TLR signaling and an overproduction of TNF- $\alpha$ after TLR stimulation in AD-HIES (7-9). Defective responses in mouse models of $\mathrm{AD}$-HIES and primary keratinocyte $(\mathrm{KC})$ and fibroblast $(\mathrm{FB})$ cultures from patients with $\mathrm{AD}$-HIES could be reversed by TNF- $\alpha$ blockade or by EMT modulators. Our results identify TNF- $\alpha$ blockade and/or EMT modulation as potential therapeutic approaches in patients with AD-HIES suffering $S$. aureus infections.

\section{Results}

An aberrant responses to $S$. aureus is associated with TNF- $\alpha$ overproduction in skin blister challenge models. Despite having colonization rates similar to those in patients with atopic dermatitis, patients with AD-HIES had significantly greater rates of $S$. aureus skin abscesses (94\% versus $10 \%)$ and S. aureus pneumonia $(42 \%$ versus 0\%; Supplemental Table 1). These data confirm reports of profound $S$. aureus susceptibility in AD-HIES that differentiates it from atopic dermatitis. To begin to assess this susceptibility, we used a suction blister model that exposes wounded skin to either autologous serum (10) or lethally irradiated bacteria in saline diluent (6). Compared with HVs, patients with AD-HIES did not display any significant deficit of IL-17A in the initially accumulated ("neat") blister fluid (Supplemental Figure 1A; 
A

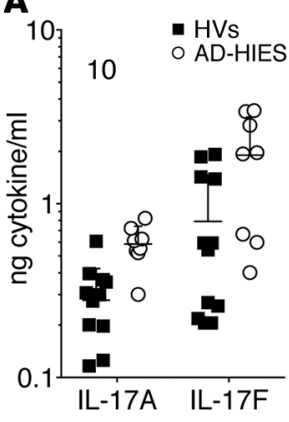

D

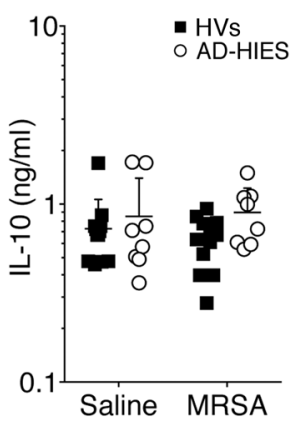

B

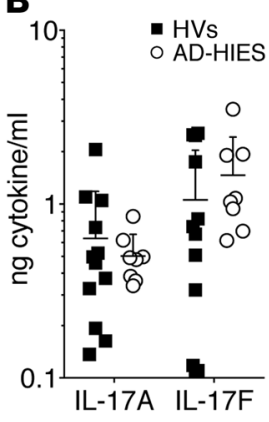

C

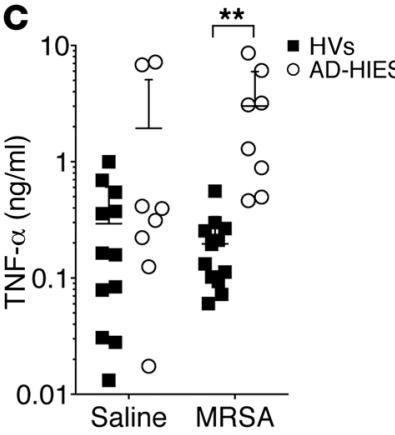

$\mathbf{E}$

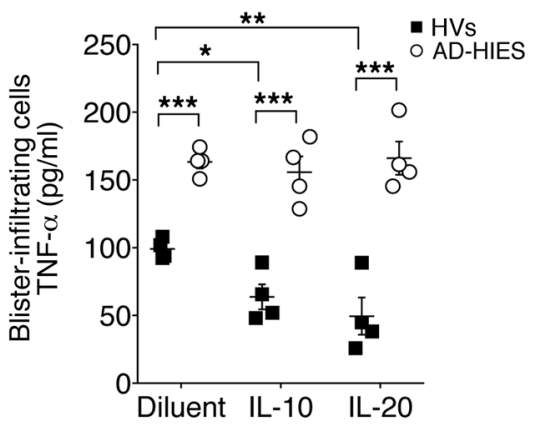

Figure 1. Human STAT3 dysfunction is associated with overproduction of TNF- $\boldsymbol{\alpha}$. (A-D) Twelve HVs and eight patients with AD-HIES underwent blister induction and challenge of the wounded area with lethally irradiated bacteria or diluent control. IL-17A, IL-17F (A and B), TNF- $\alpha$ (C), and IL-10 (D) concentrations in blister fluid after 20 hours of incubation with saline (A, C, and $\mathbf{D})$ or irradiated MRSA (B-D). (E-C) Live blister-infiltrating neutrophils $\left(1 \times 10^{5}\right)$ from the blister well challenged with irradiated MRSA as in B-D (E) or peripheral neutrophils (F) were harvested from $4 \mathrm{HVs}$ and 4 patients with AD-HIES. The cells were then incubated ex vivo for 48 hours with irradiated S. aureus (MOI of 10) and IL-10, IL-20, or diluent. (C) Approximately $1 \times 10^{6}$ primary KCs from $3 \mathrm{HVs}$ or 3 with AD-HIES patients were incubated for 24 hours with irritated MRSA as in $\mathbf{E}$ and $\mathbf{F}$. Shown is the supernatant TNF- $\alpha$ concentration from independent cell lines, which are indicated by individual dots. Data represent a combination of experiments involving individual patients $(\mathbf{A}-\mathbf{F})$ or are representative of 3 independent experiments $(\mathbf{G})$ and are displayed as the mean $\pm \mathrm{SD}$. ${ }^{*} P<0.05$, ${ }^{* *} P<0.01$, and ${ }^{* * *} P<0.001$, by ANOVA with Bonferroni's correction versus HVs across 14 tested cytokines (A-D; see also Supplemental Figure 1 and Methods).

$\mathbf{F}$

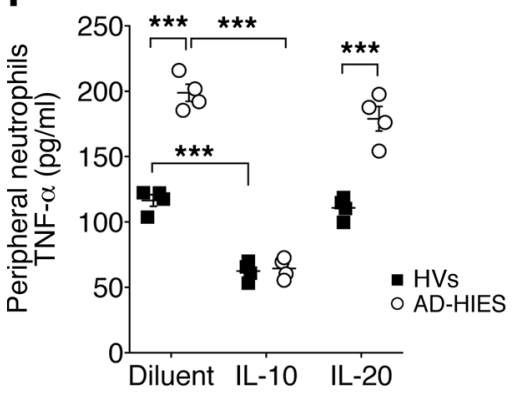

G

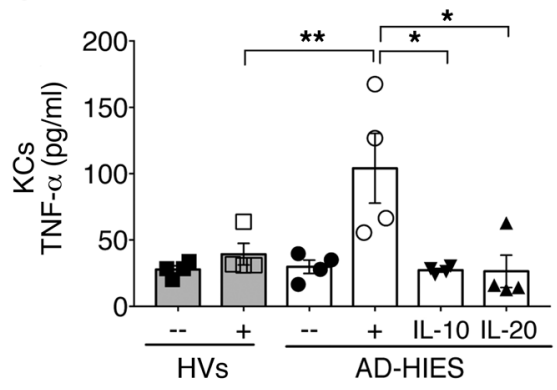

supplemental material available online with this article; https:// doi.org/10.1172/JCI121486DS1) or after exposure to 70\% autologous serum (Supplemental Figure 1B). Likewise, patients with $\mathrm{AD}$-HIES displayed no significant defect in the accumulation of IL-17A or IL-17F after exposure to saline (Figure 1A and Supplemental Table 2) or irradiated methicillin-resistant $S$. aureus (MRSA) (strain USA300 LAC) (Figure 1B and Supplemental Table 2). We performed intracellular staining on the infiltrating blister cells but were unable to identify the cellular source of IL-17. This may be indicative of technical limitations due to the predominance of neutrophils and low $\mathrm{CD}^{+} \mathrm{T}$ cell numbers in the blister fluid (Supplemental Figure 1, C and D), but may also suggest a cellular source in the underlying dermis.

Blister accumulation of other cytokines tested in response to autologous serum (Supplemental Figure 1, E and F), saline (Supplemental Figure 1G), or irradiated MRSA (Supplemental Figure 1H) did not differ between HVs and patients with AD-HIES. However, consistent with reports involving peripheral blood (11), patients had increased blister TNF- $\alpha$ concentrations in response to MRSA (Figure 1C). We observed similar but nonsignificant trends in the neat fluid and blister fluid responses to autologous serum (Supplemen- tal Figure 1I). Prior reports involving DCs suggested that STAT3 mutations may lead to overproduction of TNF- $\alpha$ as a result of the STAT3 dependence of inhibitory IL-10 signaling $(12,13)$. We found that IL-10 accumulation in the blister fluid was equivalent between HVs and patients with AD-HIES (Figure 1D). IL-10 and IL-20, a related STAT3-dependent epithelial cytokine (14), inhibited TNF- $\alpha$ production in blister-infiltrating neutrophils from HVs, but not in those from patients with AD-HIES (Figure 1E). We found that IL-10 inhibited TNF- $\alpha$ production in both HV and AD-HIES peripheral blood neutrophils (Figure 1F), suggesting differences in responsiveness resulting from activation and/or diapedesis. Peripheral neutrophils did not respond to IL-20 (Figure 1F), consistent with their reported lack of IL-20 receptor expression (15). Primary KCs from patients with AD-HIES stimulated with irradiated $S$. aureus produced more TNF- $\alpha$ than did KCs from HVs, but IL-10- and IL-20mediated inhibition was preserved (Figure 1G). Taken together, these results suggest that the TNF- $\alpha$ overproduction associated with AD-HIES may be driven by direct KC overproduction as well as failed inhibitory IL-10/IL-20 signaling in infiltrating cells.

Mouse models of AD-HIES display aberrant responses to S. aureus. We next aimed to model the aberrant responses seen in patients 
A

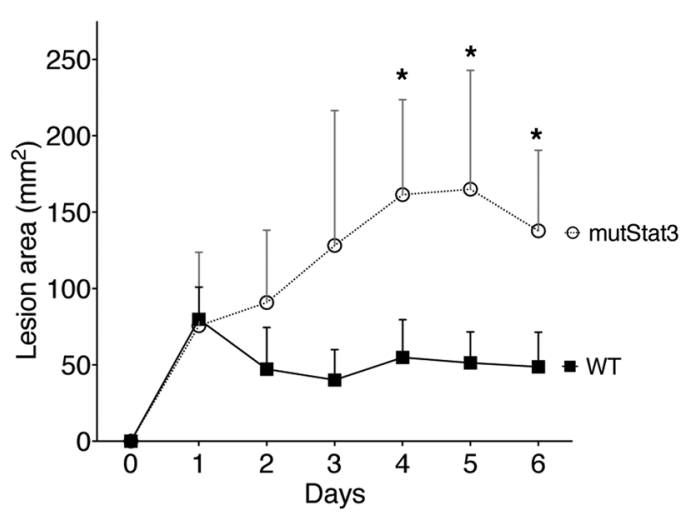

B

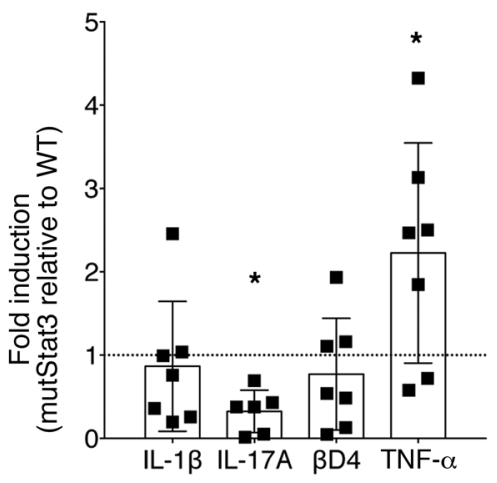

C

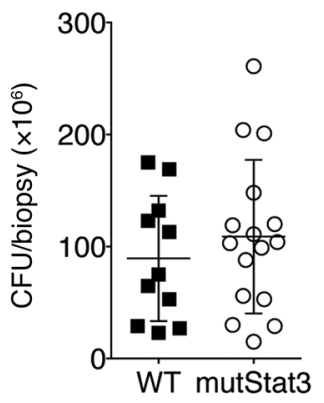

D

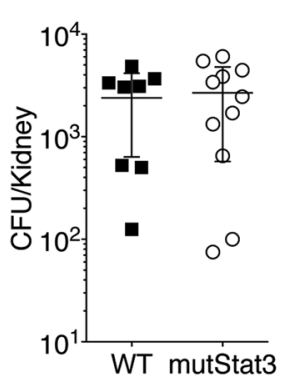

E

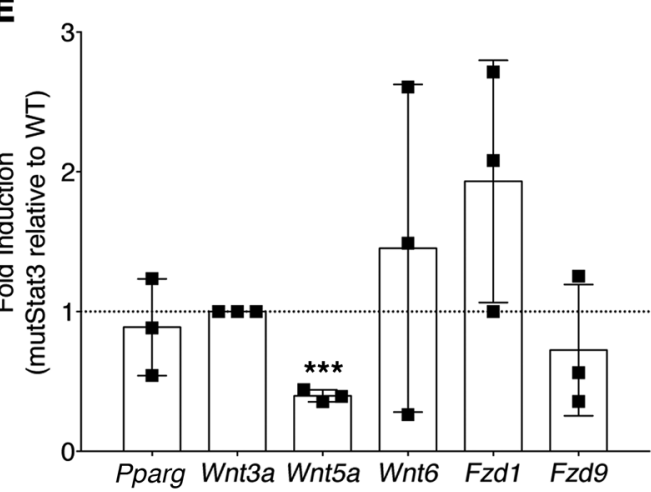

$\mathbf{F}$

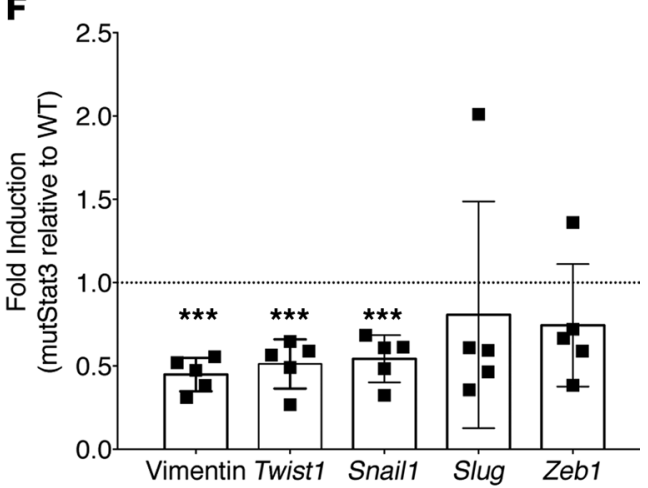

Figure 2. MutStat3 mice have a defect in their cutaneous response to S. aureus. (A) Littermate WT mice or mice with a transgenic insertion of mutated Stat3 (mutStat3) were infected with $10^{7}$ CFU MRSA by s.c. injection, and the lesion area was measured daily. (B) Transcript levels from homogenized skin harvested on day 5 or 6 in mutStat3 mice relative to WT (set to 1, dotted line), normalized to GAPDH, are shown for IL-1 $\beta$, IL-17A, and $\beta$ D4. (C) A 3-mm biopsy was taken from infected skin, and (D) the kidneys were collected on day 5 or 6 and homogenized and plated after serial dilutions to enumerate the CFU burden. (E and F) Transcript values, as in C, were normalized to GAPDH and are relative to WT. Data shown are a combination of 3 or more independent experiments and displayed as the mean \pm SD. $n=4-7$ mice per group, per experiment. ${ }^{*} P<0.05$ and ${ }^{* * *} P<0.001$, by ANOVA with Bonferroni's correction.

with AD-HIES using mutStat3 mice, which contain a transgenic insertion of 2 copies of dominant-negative Stat 3 to mimic the genotype of patients with AD-HIES (16). Consistent with the clinical presentation of AD-HIES, mutStat 3 mice had larger lesion sizes than did WT littermate mice (Figure 2A). They also had an increased abundance of TNF- $\alpha$ transcripts in $S$. aureus-infected skin (Figure 2B), similar to our human blister data. MutStat3 mice had a lower abundance of IL-17A transcripts (Figure 2B); however, bacterial numbers did not differ from those in WT mice in the skin or kidneys (a prominent site of dissemination; ref. 17 and Figure 2, $\mathrm{C}$ and $\mathrm{D}$ ), suggesting that there was no dramatic defect in bactericidal mechanisms.

We speculated that known defects in wound healing and tissue remodeling in AD-HIES (1-4), rather than direct bactericidal defects, may contribute to the aberrant responses to $S$. aureus. EMT is an essential process in wound healing that is influenced by multiple interlinked signaling pathways, including STAT3, Wnt/ $\beta$-catenin, and TNF- $\alpha$ (18-21). In line with the hypothesis of an EMT defect in AD-HIES, $S$. aureus-infected mutStat 3 mice had reduced transcript levels of Wnt5a (Figure 2E), which has been reported to inhibit TNF- $\alpha$ production from human retinal pigment epithelial cells (22). Furthermore, mutStat3 mice had reduced transcript levels of other EMT markers, including vimentin, an intermediate filament enhanced by STAT3 activation (ref. 23 and Figure $2 \mathrm{~F}$ ). Bone marrow transplantation failed to rescue lesion size defects in mutStat3 mice (Supplemental Figure 2), further suggesting potential epithelium-mediated pathology. Reduced EMT activation in AD-HIES is consistent with the clinical practice of inhibiting STAT3 as a therapeutic means of inhibiting EMT in the treatment of cancer (21). However, TNF- $\alpha$ has been described as an inducer of EMT (24), and thus evidence of reduced EMT activation in the presence of elevated TNF- $\alpha$ is an unexpected finding. This paradoxical result may reflect hormetic behaviors of high-dose TNF- $\alpha$ (25), or signaling defects specific to STAT3 mutation that lead to defective EMT $(18,19)$.

$A D$-HIES is associated with defects in cell cultures of tissue remodeling. While bone marrow transplantation failed to rescue the anti$S$. aureus response in mutStat 3 mice, we found that transplantation of mutStat3 bone marrow into WT mice worsened the outcomes (Supplemental Figure 2). Additionally, clinical bone marrow transplantation has shown variable efficacy in treating the epithelial manifestations of AD-HIES (26-30). Taken together, these data suggest that hematopoietic cells are necessary but insufficient for normal tissue responses to infection. We therefore hypothesized that dysfunction in nonhematopoietic epithelial tissue may be an independent driver of pathology. To evaluate epithelial mor- 
A

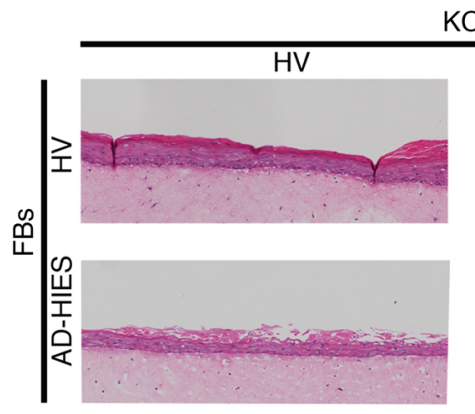

$\mathrm{KCs}$
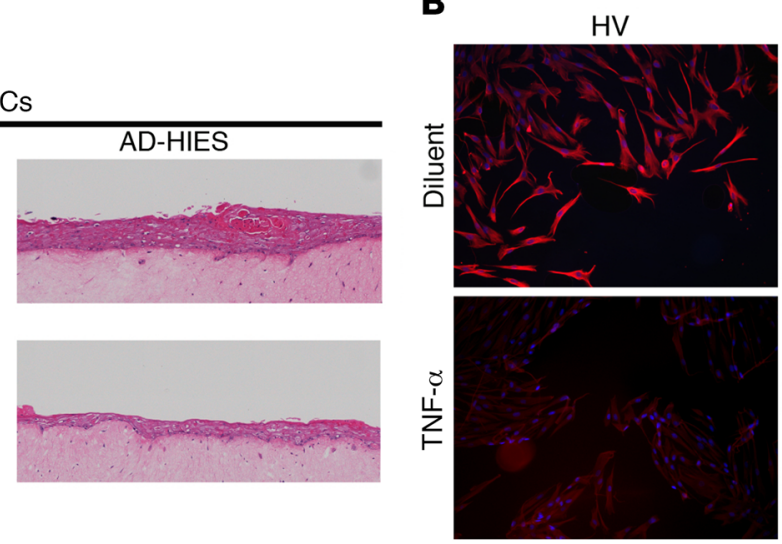

AD-HIES

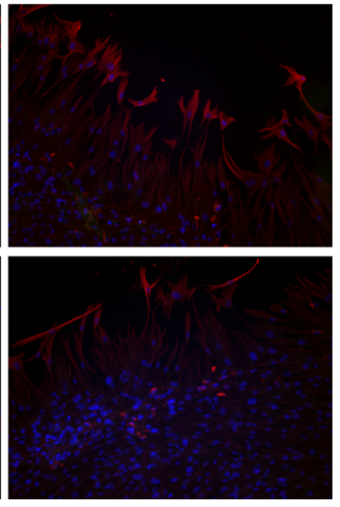

C

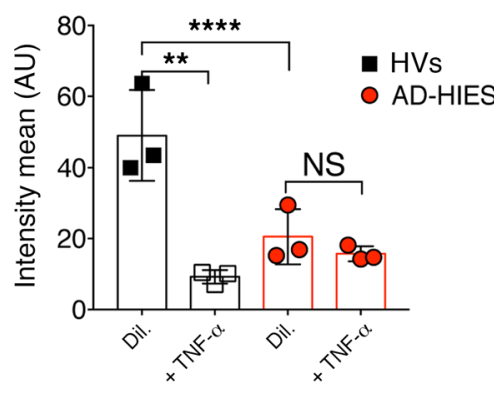

D
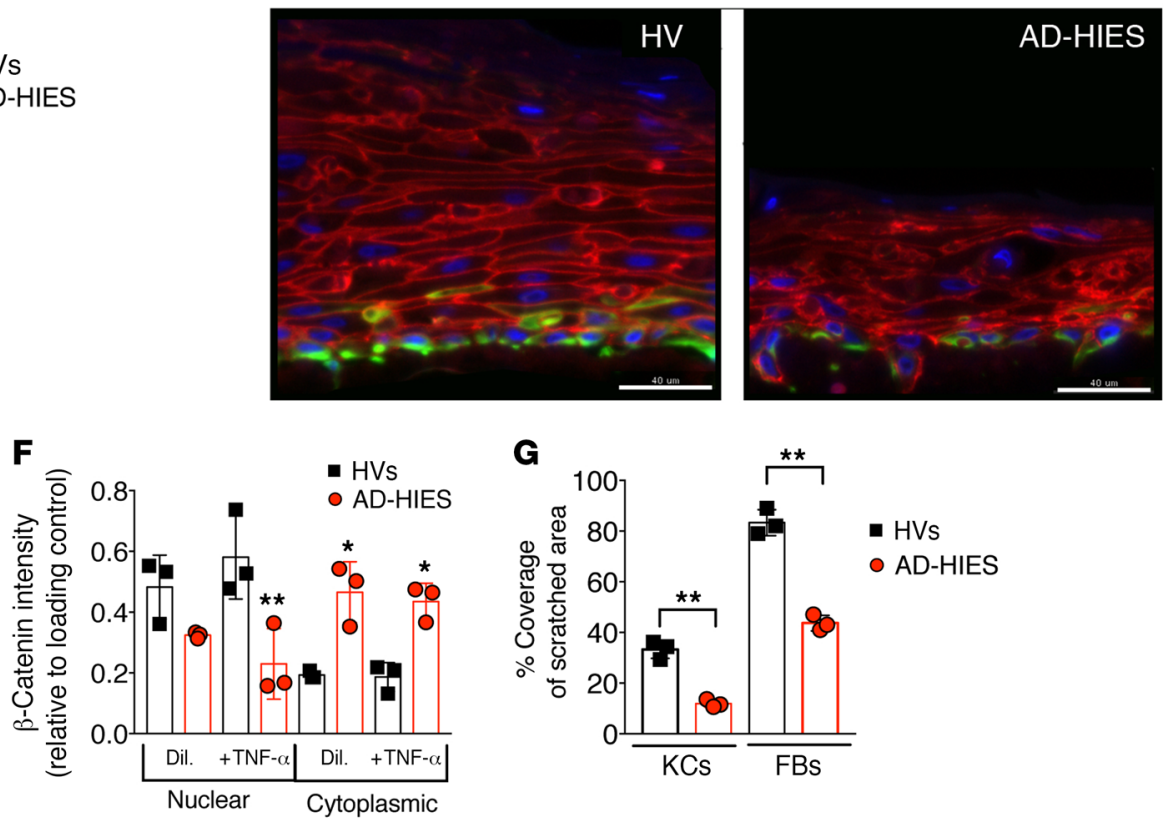

G

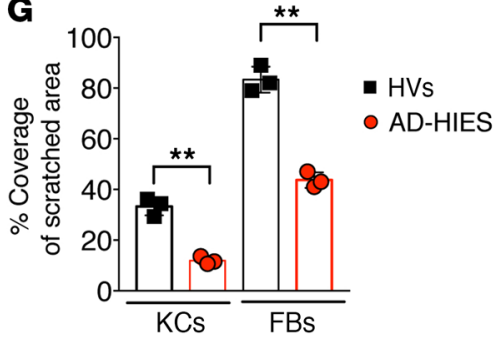

Figure 3. Skin cell cultures from patients with AD-HIES display TNF- $\alpha$-sensitive defects in wound healing. (A) 3D organotypic cultures of KCs and FBs from HVs and patients with AD-HIES. (B) IF images (original magnification, $\times 20$ ) for DAPI (blue) and vimentin (red) and (C) mean intensity of the IF signal of the leading edge of the scratch assay monolayer culture of dermal FBs from HVs and AD-HIES patients. (D) IF images of organotypic cultures, as in A, stained for $\beta$-catenin (red), vimentin (green), and DAPI (blue). FBs and KCs were sourced from both HVs and AD-HIES patients, as indicated. Scale bars: 40 $\mu \mathrm{m}$. (E) Western blot of nuclear and cytoplasmic fractions from KCs obtained from HVs and AD-HIES patients (KCs were cultured with TNF- $\alpha$ or diluent). (F) Band intensity for $\beta$-catenin, as in $\mathbf{E}$, normalized to either a histone 3 (nuclear) or $\beta$-actin (cytoplasmic) loading control. (C) Area of coverage after 18 to 22 hours of incubation in a scratch assay for KCs (see Supplemental Video 1) and FBs (see Supplemental Video 2). Data are representative of 3 or more independent experiments and displayed as the mean $\pm S D . n=3$ cell lines per group, indicated by individual dots. Each experiment used at least 2 different cell lines assayed in duplicate. ${ }^{*} P<0.05,{ }^{* *} P<0.01$, and ${ }^{* * * *} P<0.0001$, by ANOVA with Bonferroni's correction, compared with diluent treatment $(\mathbf{F})$, or as indicated. Dil., diluent.

phogenesis, we used human organotypic skin cultures formed by seeding KCs on top of FBs and allowing for self-organization into both dermal and epidermal layers (31). Compared with HV-sourced cells, organotypic cultures from AD-HIES patients failed to correctly organize into the expected epithelial layers (Figure 3A). We observed disordered organization when either KCs or FBs were sourced from patients with AD-HIES (Figure 3A).

To further characterize this disordered epithelial organization, we assessed markers of EMT in the organotypic cultures. In accordance with our mouse data (Figure $2 \mathrm{~F}$ ), vimentin signal was reduced in AD-HIES FBs, TNF- $\alpha$-treated HV FBs (Figure 3,
$\mathrm{B}$ and $\mathrm{C}$ ), and in the basal layer of AD-HIES KCs in the organotypic cultures (Figure 3D). A shift from cytoplasmic to nuclear localization of $\beta$-catenin is another marker of EMT (20). Consistent with reduced EMT activity, AD-HIES-sourced KCs showed reduced nuclear $\beta$-catenin signal (Figure 3, E and F). Similarly, AD-HIES FBs had reduced expression of the $\beta$-catenin complex N-cadherin (Supplemental Figure 3, A and B). In a scratch assay (32) model of dynamic wound healing, we further found disorganized and deficient cell healing and migration in AD-HIES-sourced KCs (Supplemental Video 1 and Figure 3G), AD-HIES-sourced FBs (Supplemental Video 2 and Figure 3G), 
A

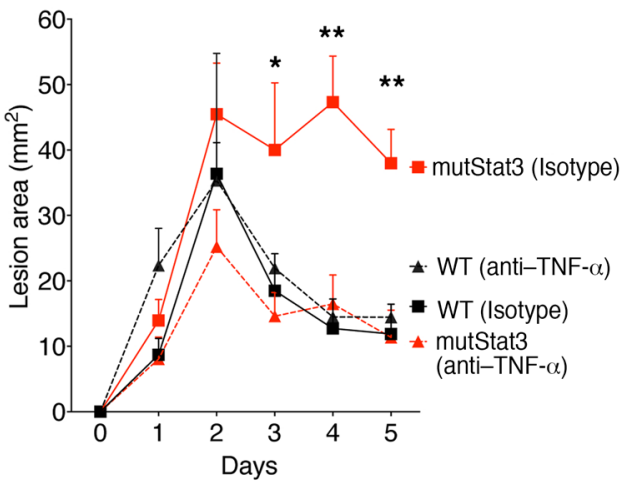

B
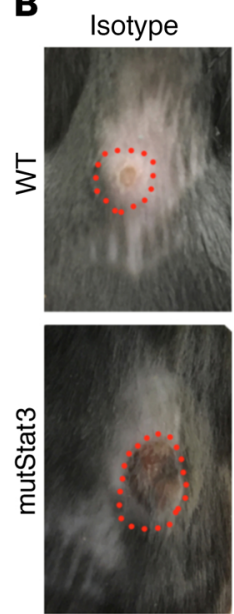

Anti-TNF- $\alpha$
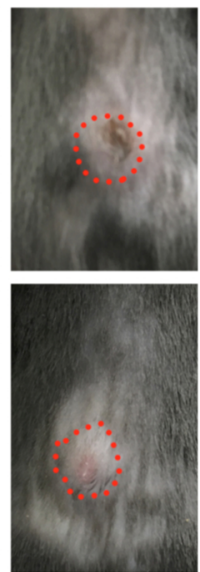

Figure 4. TNF- $\alpha$ blockade rescues aberrant antiS. aureus responses in mutStat3 mice. (A-D) Littermate WT and mutStat3 mice were treated with daily i.p. injections of anti-TNF- $\alpha$ or isotype control for 4 days prior to s.c. infection with $10^{6}$ CFU MRSA ( $n=3-5$ mice per group). The mean resultant lesion area over time (A), as well as day- 5 images of skin lesion (B), H\&E histology (original magnification, $\times 20$ ) (C), and IF staining (original magnification, $\times 10$ ) (D) from the median mouse in each group. (E and $\mathbf{F}$ ) WT and mutStat3 mice were treated with anti-TNF- $\alpha$ as above before nasal inoculation with $10^{8}$ CFU MRSA. H\&E histological images from day 7 (original magnification, $\times 40$ ) (E) and IF images (original magnification, $\times 20$ ) (F) of lung. Data are representative of 3 or more independent experiments and displayed as the mean $\pm \mathrm{SD}$. ${ }^{*} P<0.05$ and ${ }^{*} P<0.01$, by ANOVA with Bonferroni's correction versus WT diluent- or isotypetreated control.
C

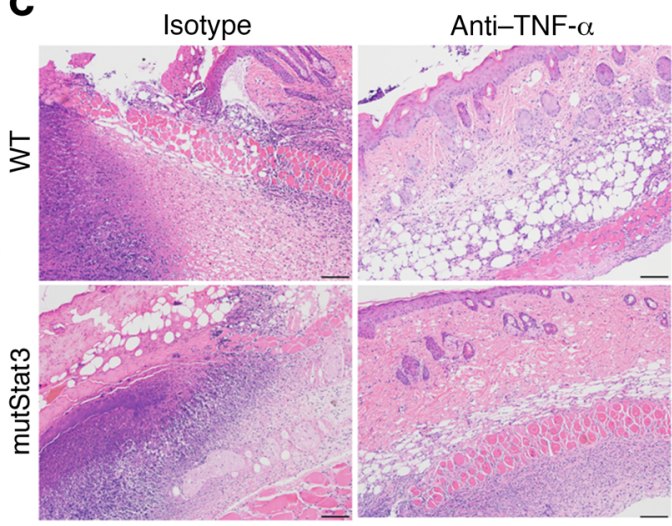

D

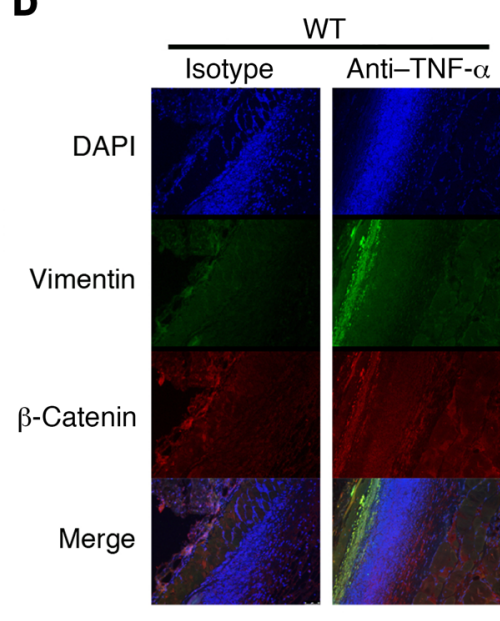

$\mathbf{F}$

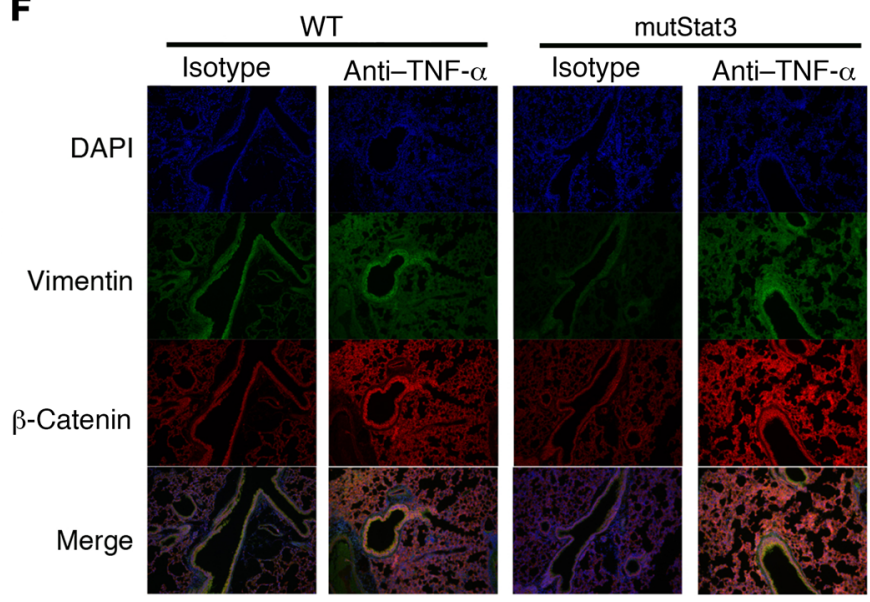

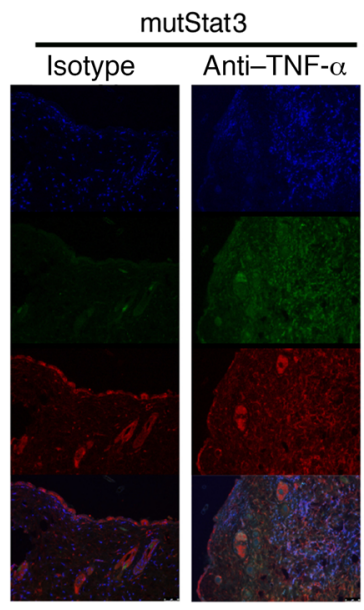

mutStat3
$\mathbf{E}$

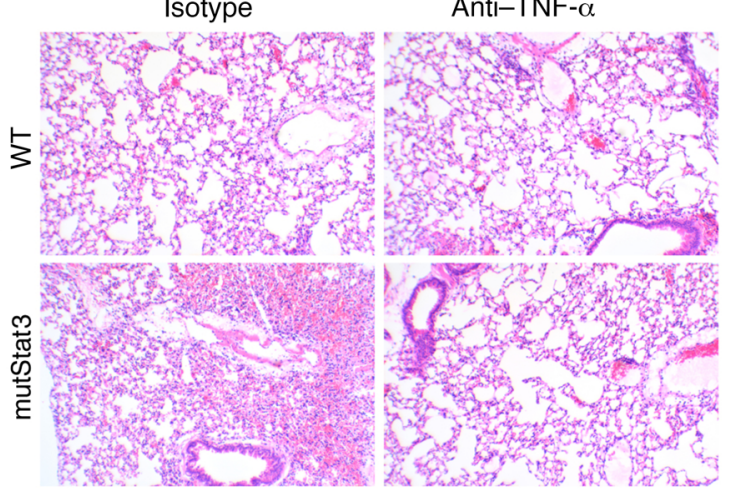

and TNF- $\alpha$-treated HV FBs (Supplemental Video 3 and Supplemental Figure 3C)

Blockade of TNF- $\alpha$ rescues aberrant anti-S. aureus responses in mutStat 3 mice. Preclinical evaluation of TNF- $\alpha$ blockade reversed pathological responses to cutaneous $S$. aureus infection in mutStat 3 mice as measured by lesion size (Figure 4, A and B); histologic evidence of epidermal inflammation (Figure $4 \mathrm{C}$ ); the expression of $\beta$-catenin and vimentin in the dermis (Figure 4D and Supplemental Figure 3D); and transcript abundance of IFN- $\gamma$ and IL-17A
(Supplemental Figure 3E). Beneficial effects were also found in a S. aureus pneumonia model, in which TNF- $\alpha$ blockade rescued mutStat3 lung pathology, characterized by multifocal and coalescing regions of intense lymphohistiocytic and eosinophilic perivascular-to-interstitial inflammation (Figure 4E) that was associated with reduced vimentin signal in the infected tissue (Figure 4F). Similar treatment effects were seen with clofazimine or rosiglitazone (Supplemental Figure 4 and Supplemental Videos 1 and 2), two unrelated drugs with reported off-target effects 
on EMT (33) that may provide alternative therapeutic strategies that avoid the potential increased risk of mycobacterial infection associated with anti-TNF- $\alpha$ therapy (34).

\section{Discussion}

Our results suggest that overexpression of TNF- $\alpha$ and disordered EMT induction may contribute to aberrant $S$. aureus responses in AD-HIES. These findings do not exclude the possibility of a contribution of IL-17 defects to $S$. aureus susceptibility. The preserved cutaneous IL-17 production seen in patients with AD-HIES should be interpreted cautiously, since we evaluated production up to 20 hours after challenge, potentially before adaptive $\mathrm{T}$ cell cytokine deficiencies may be detectable. The decreased IL-17 we observed in mutStat 3 mice 5-6 days after infection may reflect this kinetic issue or may indicate that cutaneous IL-17 production is $\gamma \delta \mathrm{T}$ cell predominant in mice versus $\alpha \beta \mathrm{T}$ cell predominant in humans (35). Our findings distinguish patients with AD-HIES from those with isolated IL-17 receptor defects or gain-of-function STAT1 mutations, in whom defective IL-17 signaling has not been associated with TNF- $\alpha$ overproduction and confers infectious susceptibilities more predominated by Candida albicans than $S$. aureus (36, 37). Furthermore, elevated TNF- $\alpha$ in the context of hypomorphic STAT3 contrasts AD-HIES with psoriasis, in which elevated cutaneous levels of TNF- $\alpha$ (38) are associated with hyperactivity of STAT3 $(39,40)$.

Failed signaling of IL-10 family cytokines in responding leukocytes appears to contribute to the observed TNF- $\alpha$ overproduction, as may other signaling defects associated with STAT3 mutations (41). Our data suggest that the defects associated with EMT pathways in dermal FBs from AD-HIES patients may be partially mediated by Wnt signaling abnormalities and potentially exacerbated by excess TNF- $\alpha$ exposure. Similarly, AD-HIES KCs show defects in cell migration and reduced acquisition of EMT markers associated with abnormal $\beta$-catenin localization. Reduced EMT activation in AD-HIES is consistent with the known anti-EMT effects of STAT3 inhibition (21) and the reported STAT3 dependence of select EMT pathways (42, 43). However, additional inquiry will be required to identify how AD-HIES mutations in STAT3 alter EMT activation.

The observed defects in EMT and $\beta$-catenin localization could conceivably affect the function of a wide variety of tissues (44), thereby contributing to the multisystem clinical spectrum seen in AD-HIES. However, tissue-specific differences in EMT pathways also indicate that extrapolation of our findings to other infection models or other diseases should be done cautiously, especially given that subtle differences in EMT markers and TNF- $\alpha$ sensitivity could be seen between FBs and KCs in our experiments.

Overall, our findings suggest that both overproduction of and paradoxical responses to TNF- $\alpha$ exposure contribute to epithelial dysfunction in patients with AD-HIES. Further mechanistic insight is needed to fully elucidate the role of STAT3 in EMT and epithelial function, but the reversal of pathology with TNF- $\alpha$ blockade and drugs with reported modulatory effects on EMT in mutStat 3 mice and AD-HIES cell culture systems represent promising preclinical findings. Additional studies will be required to assess whether these treatments can provide equivalent benefit when initiated after the onset of inflammatory pathology. Ultimately, our results suggest that abnormalities in epithelial responses, including EMT and associated TNF- $\alpha$ overproduction, may underlie the aberrant responses to $S$. aureus in patients with AD-HIES and that pharmaceutical intervention targeting these pathways may benefit this unique patient population.

\section{Methods}

Human subjects.Patients with AD-HIES and HVs were recruited to participate in this study at the NIAID under IRB-approved protocols (NCT02262819 and NCT00001257). A total of 36 patients with AD-HIES were enrolled in the studies. Under protocol NCT00001257, five AD-HIES patients and twenty-one HVs underwent suction blister induction, and, after collection of the initially accumulated (neat) blister luminal fluid and removal of the epidermis, the wounds were challenged with $70 \%$ autologous serum $(t 0)$, and the subsequently accumulated fluid was collected sixteen hours later $(t 16)$. Under NCT02262819, eight patients underwent blister induction, and the blister wounds were challenged with lethally irradiated bacteria for twenty hours prior to collection of the accumulated blister fluid; these patients also had their skin swabbed to assess $S$. aureus colonization. CFU equivalents $(2 \times$ $10^{7}$ ) of lethally irradiated $S$. aureus (strain USA300 LAC) were used for the bacterial challenge. The remaining 18 patients only had their skin swabbed to assess $S$. aureus colonization.

A total of $47 \mathrm{HVs}$ enrolled in the studies, 21 of whom underwent blister induction and challenge with $70 \%$ autologous serum (NCT00001257). An additional 12 HVs underwent blister induction, challenge with lethally irradiated bacteria, and assessment for $S$. aureus colonization (NCT02262819). The remaining 14 HVs only had their skin swabbed to assess $S$. aureus colonization. Skin bacteria isolation and culture were performed as previously described (45). Blisters were induced, and the subsequent cell infiltrate and cytokine concentrations in the blister fluid were determined as previously described $(6,46)$. Cell viability was approximately $50 \%$ in both groups as determined by LIVE/DEAD staining (BD Biosciences).

The rates of infections of $S$. aureus skin abscess and pneumonia were determined by chart review. Twenty patients with non-AD-HIES atopic dermatitis were recruited under the IRB-approved protocol NCT01164241.

Mice. Experiments were performed in both male and female mice but were age and se matched within each experiment. WT C57BL/6 mice were purchased from The Jackson Laboratory. MutStat3 mice $\left(\mathrm{C} 57 \mathrm{BL} / 6-\mathrm{Tg}\left(\mathrm{Stat}^{*}\right) 9199 \mathrm{Alau} / \mathrm{J}\right)$ were a gift from Liana Falcone (NIAID, NIH) and Josh Milner (NIAID, NIH) or were purchased from The Jackson Laboratory. Littermate control mice were used as indicated in the text. Skin infections, CFU assessment, and PCR on skin were all performed as previously described (14). Bone marrow transplantation was performed as previously described (16). For anti-TNF- $\alpha$ treatment, mice were injected i.p. with $4 \mathrm{mg} / \mathrm{kg}$ anti-TNF- $\alpha$ (R\&D Systems; catalog AB-410-NA) or goat IgG isotype (R\&D Systems) daily for 4 days. The following day, mice were challenged with $S$. aureus. Skin CFU assessment and mRNA transcript evaluations were performed as previously described (14). Mice treated with clofazimine (SigmaAldrich) and rosiglitazone (Cayman Chemical) received daily gavage beginning 3 days before and up through the day of the infection (days -3 to 0 ). Clofazimine was suspended in 1:1 DMSO and 100\% ethanol to $150 \mathrm{mg} / \mathrm{ml}$, and this stock was diluted to $7.5 \mathrm{mg} / \mathrm{ml}$ in $5 \% \mathrm{w} / \mathrm{v}$ agarose in sterile water. Rosiglitazone was suspended in 1:1 DMSO and 
$100 \%$ ethanol to $50 \mathrm{mg} / \mathrm{ml}$, and this stock was diluted to $2.5 \mathrm{mg} / \mathrm{ml}$ in sterile water. A dose of $100 \mu \mathrm{l}$ each of clofazamine and rosiglitazone was administered.

KC culture. KCs were cultured with irradiated 3T3-J2 FBs in feeder medium containing 3:1 (v/v) Ham's F-12 Nutrient Mix (Invitrogen, Thermo Fisher Scientific) containing DMEM high glucose no glutamine (Invitrogen, Thermo Fisher Scientific), 5\% newborn calf serum (Thermo Fisher Scientific), $0.4 \mu \mathrm{g} / \mathrm{ml}$ hydrocortisone (Sigma-Aldrich), 5 $\mu \mathrm{g} / \mathrm{ml}$ insulin, $8.4 \mathrm{ng} / \mathrm{ml}$ cholera toxin, $10 \mathrm{ng} / \mathrm{ml} \mathrm{EGF}$ (Invitrogen, Thermo Fisher Scientific), $24 \mu \mathrm{g} / \mathrm{ml}$ adenine, $10 \mathrm{U} / \mathrm{ml}$ penicillin and $10 \mu \mathrm{g} / \mathrm{ml}$ streptomycin, $2 \mathrm{mM}$ L-glutamine (Gibco), $100 \mu \mathrm{g} / \mathrm{ml}$ Primocin (Invitrogen), and $10 \mu \mathrm{M} \mathrm{Y-27632} \mathrm{(Tocris).} \mathrm{Y-27632} \mathrm{is} \mathrm{a} \mathrm{Rho} \mathrm{kinase} \mathrm{inhibitor,} \mathrm{and}$ its use in KC culture has been described previously $(47,48)$. 3T3-J2 feeder FBs were subcultured in DMEM $(1 \times)$ with GlutaMAX (Invitrogen, Thermo Fisher Scientific), 10\% FBS (Hyclone), $25 \mathrm{mM}$ HEPES, and $50 \mu \mathrm{g} / \mathrm{ml}$ gentamicin. FBs were cultured in $175-\mathrm{cm}^{2}$ flasks at an initial density of 2 $\times 10^{6}$ cells and passed every 3 days using $0.25 \%$ trypsin-EDTA to detach cells. Prior to coculture with the KCs, 3T3-J2 cells were $\gamma$-irradiated with a dose of $60 \mathrm{~Gy}$. Cocultured cells were passed using versene to detach the FB feeder cells, followed by $0.25 \%$ trypsin-EDTA to detach the KCs. After detachment, the KCs were passed through a 70- $\mu$ m nylon filter to break up cell aggregates and then seeded in a 10-cm dish at a density of 4 $\times 10^{5} \mathrm{KCs} / 1 \times 10^{6} 3 \mathrm{~T} 3-\mathrm{J} 2 \mathrm{FBs}$.

Primary FB culture. Primary dermal FBs were cultured in Corning $162-\mathrm{cm}^{2}$ flasks in culture medium consisting of high glucose DMEM with GlutaMAX supplement, penicillin, streptomycin, and 10\% FBS (FB DMEM complete). Once cells reached 70\%-90\% confluency, they were washed with PBS and harvested using $7 \mathrm{ml}$ of a $0.25 \%$ Trypsin/EDTA solution, before being reneutralized with $8 \mathrm{ml} \mathrm{FB} \mathrm{DMEM} \mathrm{complete} \mathrm{and}$ pelleted via centrifugation at $1,200 \mathrm{~g}$. The neutralization media were discarded before the cells were resuspended in FB DMEM complete, counted with trypan blue exclusion on a hemocytometer, and either split between flasks at a concentration of $5 \times 10^{5}$ cells $/ \mathrm{ml}$ or plated for experimental use. Cells were split and passaged every 3 to 4 days.

Organotypic raft cultures. Organotypic raft culturing was performed as previously described, with modifications (49). Primary FBs $\left(2 \times 10^{5}\right)$ from either HVs or patients with AD-HIES were seeded in $100 \%$ type $1 \mathrm{~A}$ rat-tail collagen (Corning) with $2 \times 10^{5}$ primary KCs from either HVs or patients with AD-HIES and seeded in a 24-well cell culture plate with 1 ml raft medium (3:1 Ham's F12 Nutrient Mix, Invitrogen, Thermo Fisher Scientific) containing DMEM High Glucose No Glutamine (Invitrogen, Thermo Fisher Scientific), 5\% newborn calf serum (Thermo Fisher Scientific), $0.4 \mu \mathrm{g} / \mathrm{ml}$ hydrocortisone (Sigma-Aldrich), $10 \mathrm{ng} / \mathrm{ml}$ human EGF (Invitrogen, Thermo Fisher Scientific), $0.1 \mathrm{nM}$ cholera toxin, $5 \mu \mathrm{g} /$ $\mathrm{ml}$ insulin, $2 \mathrm{mM}$ glutamine (Gibco, Thermo Fisher Scientific), and 100 $\mu \mathrm{g} / \mathrm{ml}$ Primocin (Invitrogen, Thermo Fisher Scientific) and incubated overnight at $37^{\circ} \mathrm{C}$ and $5 \% \mathrm{CO}_{2}$. The next day, the rafts were lifted onto a stainless-steel metal mesh and incubated in 6-well cell plates at the liquid-air interface for 13 days. Approximately $3 \mathrm{ml}$ raft medium was used for each raft and changed every 2 days. After 13 days, the rafts were washed with PBS, fixed in formalin, and processed as described in the Immunofluorescence microscopy section of Methods.

$K C$ and FB cytokine induction assays. Primary KCs were seeded in 2 types of media for these assays: $\operatorname{SFM}(+)$ containing Serum-Free Medium (SFM) (Gibco, Thermo Fisher Scientific) supplemented with $10 \mathrm{ng} / \mathrm{ml} \mathrm{EGF,} 50 \mu \mathrm{g} / \mathrm{ml}$ Bovine Pituitary Extract (BPE), $100 \mu \mathrm{g} /$ $\mathrm{ml}$ Primocin, and $10 \mu \mathrm{M}$ Y-27636; or SFM(-), containing SFM with
$50 \mu \mathrm{g} / \mathrm{ml} \mathrm{BPE}$ and $100 \mu \mathrm{g} / \mathrm{ml}$ Primocin. On day 0, cells were seeded in $1 \mathrm{ml} \mathrm{SFM(+)} \mathrm{at} \mathrm{a} \mathrm{density} \mathrm{of} 2 \times 10^{5}$ cells/well in a 24 -well cell culture plate and incubated at $37^{\circ} \mathrm{C}$ with $5 \% \mathrm{CO}_{2}$ for 24 hours. On day 1 , cells were washed 3 times with PBS, incubated briefly, agitated with $0.5 \mathrm{ml}$ versene, and washed again 3 times with PBS followed by addition of $1 \mathrm{ml} \mathrm{SFM(-).} \mathrm{Cells} \mathrm{were} \mathrm{then} \mathrm{incubated} \mathrm{again} \mathrm{at} 37^{\circ} \mathrm{C}$ and $5 \%$ $\mathrm{CO}_{2}$ for 24 hours. On day 2, cells were first washed with PBS 3 times, and then $400 \mu \mathrm{l} \mathrm{SFM(-)} \mathrm{containing} \mathrm{the} \mathrm{treatment} \mathrm{was} \mathrm{added.} \mathrm{For} \mathrm{FBs,}$ on day 0 , cells were seeded in $1 \mathrm{ml}$ FB DMEM complete at a density of $2 \times 10^{5}$ cells/well in a 24 -well cell culture plate and incubated at $37^{\circ} \mathrm{C}$ with $5 \% \mathrm{CO}_{2}$ for 24 hours. On day 1 , the $\mathrm{FBs}$ were washed with PBS and incubated in DMEM with GlutaMAX supplement, penicillin, and streptomycin only (FB SFM) for another 24 hours at $37^{\circ} \mathrm{C}$ and $5 \%$ $\mathrm{CO}_{2}$ for 24 hours. On day 2, cells were washed again with PBS and then received $400 \mu \mathrm{l} \mathrm{FB} \mathrm{SFM-containing} \mathrm{treatments.} \mathrm{For} \mathrm{the} \mathrm{human} \beta$ defensin induction assay, the treatments were a combination of $10 \mathrm{ng} /$ $\mathrm{ml}$ IL-17A (R\&D Systems) and IFN- $\gamma$ (R\&D Systems) or 0.1\% BSA in PBS (no-treatment control). For the $\beta$-catenin and vimentin induction assay, the treatments were $2 \mathrm{ng} / \mathrm{ml} \mathrm{TNF-} \alpha$ (R\&D Systems) or $0.1 \%$ BSA in PBS. Cells were incubated at $37^{\circ} \mathrm{C}$ and $5 \% \mathrm{CO}_{2}$ for 48 hours, after which RNA, protein, or supernatant was collected.

Immunoblot analysis. Protein was isolated using the NE-PER Kit (Thermo Fisher Scientific) following the manufacturer's instructions. Protein amounts were quantified using the Pierce BCA assay (Thermo Fisher Scientific). Protein ( $2 \mu \mathrm{g} / \mathrm{sample}$ ) was loaded into a $1.5-\mathrm{mm}$, 10-well NuPAGE 4\%-12\% Bis-Tris Gel (Thermo Fisher Scientific) and imaged using an Odyssey 3034 (LI-COR Biosciences). Integrated densitometry was calculated using ImageJ software (NIH). The following antibodies were used: $\beta$-catenin (E-5, Santa Cruz Biotechnology, catalog sc-7964; 1:50); vimentin (Life Technologies, Thermo Fisher Scientific, catalog MA5-11883; 1:50); histone H3 (D1H2, Cell Signaling Technology, catalog 44996; 1:500); $\beta$-actin (13E5, Cell Signaling Technology, catalog 4970S; 1:1,000); Rabbit IgG (H\&L) Antibody DyLight 800 Conjugated (Rockland, catalog 611-145-002; 1:10,000); and Mouse IgG (H\&L) Antibody DyLight 680 Conjugated (Rockland Antibodies and Assays, catalog 610-444-002).

Real-time relative reverse transcription PCR. Real-time relative reverse transcription PCR (RT-PCR) was performed using the Applied Biosystems 7500 Fast Real-time PCR system as previously described (14). The TaqMan One-Step Real-Time RT-PCR Kit (Thermo Fisher Scientific) was used to detect mRNA expression of human GAPDH (Thermo Fisher Scientific, HsO2758991_g1) and hBD2 (Thermo Fisher Scientific, Hs04194486_g1). Mouse primers were also purchased from Thermo Fisher Scientific as described previously (14), with additional markers including Pparg (Mm00440940_m1); Wnt3a (Mm00437337_ m1); Wht5a (Mm00437347_m1); Wht6 (Mm00437353_m1); Fzd1 (Mm00445405_s1);Fzd9 (Mm01206511_s1); vimentin (Mm00619195_ g1); Twist1 (Mm00442036_m1); Snail1 (Mm00441533_g1); Slug (Mm00441531_m1); and Zeb1 (Mm00495564_m1).

ELISA. hBD2 was quantified using the Human BD2 Standard TMB ELISA Development Kit (PeproTech) according to the manufacturer's instructions, with minor alterations. A streptavidin concentration of $0.5 \mu \mathrm{g} / \mathrm{ml}$ was used, and the color was allowed to develop for 10 minutes after addition of tetramethylbenzidine (TMB). Cytokine concentrations for Supplemental Figure 1, A, B, E, and F were determined using custom plates for the indicated analytes (Meso Scale Diagnostics). 
Fluorescence microscopy scratch assay. Coverslips (15-mm) were placed inside each well of two 24-well plates, one designated $t 0$ and the other $t 18$. KCs were seeded at a density of $2 \times 10^{5}$ cells/well in SFM $(+)$ at $37^{\circ} \mathrm{C}$. After 24 hours, the media were replaced with SFM without EGF and Y-27632 SFM(-), and for those media that received $2 \mu \mathrm{g} / \mathrm{ml}$ clofazimine (Sigma-Aldrich) and $3.5 \mu \mathrm{g} / \mathrm{ml}$ rosiglitazone (Cayman Chemical) in advance of TNF- $\alpha$ (R\&D Systems), the supplements were added at this point, and then both plates were incubated for another 24 hours at $37^{\circ} \mathrm{C}$. The next day, the cells were washed with PBS and the media replaced with SFM(-) containing the appropriate amounts of the following: $2 \mu \mathrm{g} / \mathrm{ml}$ clofazimine, $3.5 \mu \mathrm{g} / \mathrm{ml}$ rosiglitazone, $20 \mathrm{ng} / \mathrm{ml} \mathrm{TNF-} \alpha$, or $0.05 \%$ ethanol to serve as the diluent control. The cells were incubated for another 24 hours at $37^{\circ} \mathrm{C}$, and the next day, the cells were washed with PBS and $0.5 \mathrm{ml}$ of $10 \mu \mathrm{g} / \mathrm{ml}$ mitomycin C for 2 hours at $37^{\circ} \mathrm{C}$. Next, the media were replaced with PBS, and using a 200- $\mu$ l RAININ pipette tip, a scratch was made lengthwise down the well spanning the entire diameter. The cells were then washed 3 times with PBS, and 3.7\% formaldehyde in PBS was added to the plate designated $t 0 . \mathrm{SFM}(-)$ media were added to the $t 18$ plate and placed back into the $37^{\circ} \mathrm{C}$ incubator for 18 hours. Following this, the media were aspirated and replaced with $3.7 \%$ formaldehyde in PBS. For FBs, the same general procedure was followed, except the cells were seeded in FB DMEM complete media for 24 hours, which was then replaced with DMEM complete containing each treatment supplemented in the media.

The immunostaining procedure began by washing the coverslips in PBS, followed by permeabilization for 5 minutes in $0.1 \%$ Triton $\mathrm{X}-100$ in PBS. Cells were then blocked for 1 hour at room temperature in $3 \%$ BSA with $1 \%$ FBS and $0.1 \%$ Tween-20 in PBS, followed by incubation overnight at $4^{\circ} \mathrm{C}$ with a primary antibody for $\beta$-catenin (Santa Cruz Biotechnology, catalog sc-7963) or vimentin (Life Technologies, Thermo Fisher Scientific, catalog MA5-11883; 1:50). The next day, the cells were washed in $0.05 \%$ Tween-20 in PBS and then incubated for 1 hour at room temperature with the secondary antibody goat anti-mouse IgG AF594 (Thermo Fisher Scientific, catalog A-11005). The cells were then washed again in the $0.05 \%$ Tween-20/PBS mixture and then incubated with $300 \mathrm{nM}$ DAPI (Thermo Fisher Scientific) for 5 minutes. The slides were mounted using Thermo PermaFluor and imaged using a Leica DMI6000 Inverted Fluorescence Microscope equipped with a Leica DFC345 Monochrome camera and using LAS X software for data collection (original magnifications of $\times 10$ and $\times 63$ were used).

Percentage of coverage determined by fluorescence: DAPIstained cells within an area measuring $838 \times 698 \mu \mathrm{M}^{2}$ in each image were counted using Imaris 9.0. The percentage of coverage was determined as follows:

$\mathrm{T}_{18}=$ Average cell number from hour-18 slide scratch area $(n=5)$

$\mathrm{T}_{0}=$ Average cell number from hour-0 slide scratch area $(n=5)$

$\mathrm{T}_{\mathrm{Avg}}=$ Average cell number from hour-18 slide nonscratch area $(n=3)$

$$
\left(\frac{T_{18}-T_{0}}{T_{\text {Avg }}}\right) \times 100
$$

(Equation 1)

Light microscopy scratch assay. The light microscopy scratch assay was performed similarly to the fluorescence assay, except that only single plates were used instead of duplicate plates. Before the cells are seeded, the 24 -well plate was prepared by etching a line on the bottom of the plate across the width of each well $(0.5 \mathrm{~cm}$ down from the top of the well) to provide a reference point for subsequent images. Images were taken with a Zeiss Axiovert 200 using an exposure time of $700 \mathrm{~ms}$ just after the scratch was made at $t 0$, and then again after 18 hours of $37^{\circ} \mathrm{C}$ incubation. The original magnification used to image the cells was $\times 2.5$.

Live imaging scratch assay. The setup and treatment concentrations were the same as those described in the fluorescence microscopy scratch assay, except that only one 24-well plate was used instead of two. After the scratch was made, the cells were placed in a container on a movable stage that was kept at $37^{\circ} \mathrm{C}$ and $5 \% \mathrm{CO}_{2}$. Two positions were programmed for each well using the LAS X program, and a $\times 10$ image was taken every 10 minutes for 22 hours. The images were compiled into a movie using Imaris 9.0, which was also used to calculate the percentage of wound healing. The total surface area of the scratch at both the beginning and the end of the 22-hour period was measured using the "insert surface" feature. Then, the 22-hour surface area was divided by the 0 -hour surface area, which gave the percentage of change in wound area. This change in wound area was subtracted from $100 \%$ to give the relative percentage of wound healing in each condition for each cell line.

Immunofluorescence microscopy of paraffin-embedded tissue. Tissue sections were fixed in formalin and sent to Histoserv Inc. for processing. Sections were deparaffinized with three 3-minute washes with Xylene substitute (Thermo Fisher Scientific, catalog 9990505). Following this, tissue was washed with three separate 3-minute washes in 100\% dehydrant (Thermo Fisher Scientific, catalog 22050106), and then one 3-minute wash in 95\% dehydrant (Thermo Fisher Scientific, catalog 22050107). Tissue was then rinsed with tap water and submerged in deionized water briefly, heat-induced antigen retrieval was performed on the tissue using epitope retrieval solution, $\mathrm{pH} 6$ (Leica, catalog RE7113-CE), and an Instant Pot pressure cooker was set on "soup" at high pressure for 40 minutes. Slides were then washed in PBS for 5 minutes, permeabilized with $0.1 \%$ Triton X-100 in PBS for 10 minutes, and blocked in 3\% BSA with $1 \%$ FBS and 0.1\% Tween-20 in PBS for 2 hours at room temperature. Next, the primary antibody targeting $\beta$-catenin (Santa Cruz Biotechnology, catalog sc-7963) was added at a concentration of $4 \mu \mathrm{g} / \mathrm{ml}$ blocking solution and incubated overnight at $4^{\circ} \mathrm{C}$. Following this, the slides were washed 3 times with $0.1 \%$ Tween-20 in PBS, and the secondary antibody goat anti-mouse IgG AF594 (Thermo Fisher Scientific, catalog A-11005) was added at a concentration of $10 \mu \mathrm{g} / \mathrm{ml}$ in the blocking solution and incubated at room temperature for 2 hours. DAPI was added at a concentration of $300 \mathrm{nM}$ for 10 minutes, and following this, the slides were washed again 3 times with.1\% Tween-20 in PBS and then mounted using Lab Vision PermaFluor (Thermo Fisher Scientific). The slides were imaged as described in the Fluorescence microscopy scratch assay section of Methods.

Statistics. Means were compared by unpaired, 2-tailed $t$ test or by ANOVA for comparisons of multiple samples, using GraphPad Prism (GraphPad Software). Bonferroni's correction for Figure 2, A-C, accounted for the 14 analytes in the multiplex assay. A $P$ value of less than 0.05 was considered statistically significant.

Study approval. Studies in humans were conducted under the registered clinical trials NCT02262819 and NCT00001257 (www.clinicaltrials.gov) after approval from the IRB of the NIAID, NIH. Patients with non-AD-HIES atopic dermatitis were recruited under the NIAID IRB-approved protocol NCT01164241. All subjects 
provided informed consent prior to their participation in the study. All murine experiments were performed in compliance with the guidelines of the IACUC of the NIAID, NIH.

\section{Author contributions}

IAM designed the studies, performed and analyzed experiments, and wrote the manuscript. EDA and NJE designed, performed, and analyzed select experiments and wrote the manuscript. KAZ designed and conducted experiments involving autologous serum wound challenge. IS designed and performed experiments related to KC cultures. KWW evaluated patients and assisted in the collection of clinical samples. PG conducted flow cytometry on blister-infiltrating cells. INM performed pathologic evaluations of all H\&E-stained samples. SG assisted with immunofluorescence (IF) staining and imaging. CJF designed and performed select mouse infection experiments. AL assisted with mutStat 3 mice experiments and performed bone marrow transplantations. MG performed wound challenges involving autologous serum. DBK performed cytokine analysis on samples challenged with autologous serum. MDK and AS assisted with select experiments. PAW and DAD provided administrative support for all patients and HVs enrolled in the protocol. JIG, AFF, and SMH provided clinical supervision for all patients. SKD oversaw the project and wrote the manuscript. All authors critically reviewed the manuscript.

\section{Acknowledgments}

We would like to thank the patients, their families, and the healthy participants for their assistance. We thank the NIAID veterinarians and animal care and breeder technicians, as well as U. Topolino and $\mathrm{N}$. Topolino (NIAID) for their cooperation and sacrifice during the course of this project. This work was supported by the Intramural Research Program of the NIAID and the NIH. This project was also funded in part with federal funds from the National Cancer Institute (NCI), NIH (contract HHSN261200800001E). The content of this publication does not necessarily reflect the views or policies of the Department of Health and Human Services, nor does mention of commercial products imply endorsement by the US Government.

Address correspondence to: Ian A. Myles, 9000 Rockville Pike, Building 10, Room 11S253, Bethesda, Maryland 20892, USA. Phone: 301.451.8420; Email: mylesi@niaid.nih.gov.
1. Farmand S, Sundin M. Hyper-IgE syndromes: recent advances in pathogenesis, diagnostics and clinical care. Curr Opin Hematol. 2015;22(1):12-22.

2. Freeman AF, Olivier KN. Hyper-IgE syndromes and the lung. Clin Chest Med. 2016;37(3):557-567.

3. Freeman AF, Holland SM. Clinical manifestations, etiology, and pathogenesis of the hyper-IgE syndromes. Pediatr Res. 2009;65 (5 pt 2):32R-37R.

4. Freeman AF, et al. Lung parenchyma surgery in autosomal dominant hyper-IgE syndrome. J Clin Immunol. 2013;33(5):896-902.

5. Milner JD, et al. Impaired T(H)17 cell differentiation in subjects with autosomal dominant hyperIgE syndrome. Nature. 2008;452(7188):773-776.

6. Myles IA, et al. Transplantation of human skin microbiota in models of atopic dermatitis. JCI Insight. 2016;1(10):e86955.

7. Renner ED, et al. No indication for a defect in tolllike receptor signaling in patients with hyper-IgE syndrome. J Clin Immunol. 2005;25(4):321-328.

8. Hawn TR, et al. Hyper-IgE syndrome is not associated with defects in several candidate toll-like receptor pathway genes. Hum Immunol. 2005;66(7):842-847.

9. Yeganeh $\mathrm{M}$, et al. Toll-like receptor stimulation induces higher TNF-alpha secretion in peripheral blood mononuclear cells from patients with hyper IgE syndrome. Int Arch Allergy Immunol. 2008;146(3):190-194.

10. Hsu AP, et al. Intermediate phenotypes in patients with autosomal dominant hyper-IgE syndrome caused by somatic mosaicism. JAllergy Clin Immunol. 2013;131(6):1586-1593.

11. Holland SM, et al. STAT3 mutations in the hyper-IgE syndrome. $N$ Engl J Med. 2007;357(16):1608-1619.

12. Saito M, et al. Defective IL-10 signaling in hyperIgE syndrome results in impaired generation of tolerogenic dendritic cells and induced regulatory T cells. J Exp Med. 2011;208(2):235-249.
13. Freeman AF, Holland SM. The hyper-IgE syndromes. Immunol Allergy Clin North Am. 2008;28(2):277-291, viii.

14. Myles IA, et al. Signaling via the IL-20 receptor inhibits cutaneous production of IL- $1 \beta$ and IL-17A to promote infection with methicillin-resistant Staphylococcus aureus. Nat Immunol. 2013;14(8):804-811.

15. Gough P, Ganesan S, Datta SK. IL-20 signaling in activated human neutrophils inhibits neutrophil migration and function. JImmunol. 2017;198(11):4373-4382.

16. Steward-Tharp SM, et al. A mouse model of HIES reveals pro- and anti-inflammatory functions of STAT3. Blood. 2014;123(19):2978-2987.

17. Hahn BL, Onunkwo CC, Watts CJ, Sohnle PG. Systemic dissemination and cutaneous damage in a mouse model of staphylococcal skin infections. Microb Pathog. 2009;47(1):16-23.

18. Chen $\mathrm{G}$, et al. TNF- $\alpha$-inducing protein of Helicobacter pylori induces epithelial-mesenchymal transition (EMT) in gastric cancer cells through activation of IL-6/STAT3 signaling pathway. Biochem Biophys Res Commun. 2017;484(2):311-317.

19. Li CW, et al. Epithelial-mesenchymal transition induced by TNF- $\alpha$ requires NF- $\kappa \mathrm{B}$-mediated transcriptional upregulation of Twist1. Cancer Res. 2012;72(5):1290-1300.

20. Kalluri R, Weinberg RA. The basics of epithelial-mesenchymal transition. J Clin Invest. 2009;119(6):1420-1428.

21. Wendt MK, Balanis N, Carlin CR, Schiemann WP. STAT3 and epithelial-mesenchymal transitions in carcinomas. JAKSTAT. 2014;3(1):e28975.

22. Kim JH, Park S, Chung H, Oh S. Wnt5a attenuates the pathogenic effects of the Wnt/ $\beta$-catenin pathway in human retinal pigment epithelial cells via down-regulating $\beta$-catenin and Snail. $B M B$ Rep. 2015;48(9):525-530.

23. Wu Y, Diab I, Zhang X, Izmailova ES, Zehner ZE. Stat 3 enhances vimentin gene expression by binding to the antisilencer element and interacting with the repressor protein, ZBP-89. Oncogene. 2004;23(1):168-178.

24. Yan C, et al. Epithelial to mesenchymal transition in human skin wound healing is induced by tumor necrosis factor- $\alpha$ through bone morphogenic protein-2. Am J Pathol. 2010;176(5):2247-2258.

25 . Kraig RP, et al. TNF- $\alpha$ and microglial hormetic involvement in Neurological Health \& Migraine. Dose Response. 2010;8(4):389-413.

26. Gennery AR, Flood TJ, Abinun M, Cant AJ. Bone marrow transplantation does not correct the hyper IgE syndrome. Bone Marrow Transplant. 2000;25(12):1303-1305.

27. Dimitrova D, Freeman AF, Holland SM, Gea-Banacloche J, Kanakry CG, Kanakry JA. Allogeneic bone marrow transplantation for STAT3 deficiency. Biol Blood Marrow Transplant. 2017;23(3):S267-S268.

28. Goussetis E, et al. Successful long-term immunologic reconstitution by allogeneic hematopoietic stem cell transplantation cures patients with autosomal dominant hyper-IgE syndrome. J Allergy Clin Immunol. 2010;126(2):392-394.

29. Patel NC, Gallagher JL, Torgerson TR, Gilman AL. Successful haploidentical donor hematopoietic stem cell transplant and restoration of STAT3 function in an adolescent with autosomal dominant hyper-IgE syndrome. J Clin Immunol. 2015;35(5):479-485.

30. Yanagimachi M, et al. The potential and limits of hematopoietic stem cell transplantation for the treatment of autosomal dominant hyper-IgE syndrome. J Clin Immunol. 2016;36(5):511-516.

31. Shamir ER, Ewald AJ. Three-dimensional organotypic culture: experimental models of mammalian biology and disease. Nat Rev Mol Cell Biol. 2014;15(10):647-664.

32. Stojadinovic $\mathrm{O}$, et al. Molecular pathogenesis of chronic wounds: the role of $\beta$-catenin and c-myc in the inhibition of epithelialization and wound 
healing. Am J Pathol. 2005;167(1):59-69.

33. Koval AV, et al. Anti-leprosy drug clofazimine inhibits growth of triple-negative breast cancer cells via inhibition of canonical Wnt signaling. Biochem Pharmacol. 2014;87(4):571-578.

34. Melia E, Freeman AF, Shea YR, Hsu AP, Holland SM, Olivier KN. Pulmonary nontuberculous mycobacterial infections in hyper-IgE syndrome. J Allergy Clin Immunol. 2009;124(3):617-618.

35. Cho JS, et al. IL-17 is essential for host defense against cutaneous Staphylococcus aureus infection in mice. JClin Invest. 2010;120(5):1762-1773.

36. Puel A, et al. Chronic mucocutaneous candidiasis in humans with inborn errors of interleukin-17 immunity. Science. 2011;332(6025):65-68.

37. Toubiana J, et al. Heterozygous STAT1 gainof-function mutations underlie an unexpectedly broad clinical phenotype. Blood. 2016;127(25):3154-3164.

38. Mease PJ. Tumour necrosis factor (TNF) in psoriatic arthritis: pathophysiology and treat- ment with TNF inhibitors. Ann Rheum Dis. 2002;61(4):298-304.

39. Calautti E, Avalle L, Poli V. Psoriasis: a STAT3-centric view. Int J Mol Sci. 2018;19(1):E171.

40. Yang L, Li B, Dang E, Jin L, Fan X, Wang G. Impaired function of regulatory $\mathrm{T}$ cells in patients with psoriasis is mediated by phosphorylation of STAT3. J Dermatol Sci. 2016;81(2):85-92.

41. Wan CK, et al. Opposing roles of STAT1 and STAT3 in IL-21 function in CD4 ${ }^{+} \mathrm{T}$ cells. Proc Natl Acad Sci U S A. 2015;112(30):9394-9399.

42. Xiao J, et al. IL-6 promotes epithelial-to-mesenchymal transition of human peritoneal mesothelial cells possibly through the JAK2/STAT3 signaling pathway. Am J Physiol Renal Physiol. 2017;313(2):F310-F318.

43. Renjini AP, Titus S, Narayan P, Murali M, Jha RK, Laloraya M. STAT3 and MCL-1 associate to cause a mesenchymal epithelial transition. J Cell Sci. 2014;127(pt 8):1738-1750.

44. Clevers $\mathrm{H}$. Wnt/ $\beta$-catenin signaling in develop- ment and disease. Cell. 2006;127(3):469-480.

45. Myles IA, Reckhow JD, Williams KW, Sastalla I, Frank KM, Datta SK. A method for culturing Gram-negative skin microbiota. BMC Microbiol. 2016;16:60.

46. Gough P, Ganesan S, Datta SK. IL-20 signaling in activated human neutrophils inhibits neutrophil migration and function. J Immunol. 2017;198(11):4373-4382.

47. Chapman S, Liu X, Meyers C, Schlegel R, McBride AA. Human keratinocytes are efficiently immortalized by a Rho kinase inhibitor. JClin Invest. 2010;120(7):2619-2626.

48. Chapman S, McDermott DH, Shen K, Jang MK, McBride AA. The effect of Rho kinase inhibition on long-term keratinocyte proliferation is rapid and conditional. Stem Cell Res Ther. 2014;5(2):60.

49. Banerjee NS, Chow LT, Broker TR. Retrovirusmediated gene transfer to analyze HPV gene regulation and protein functions in organotypic "raft" cultures. Methods Mol Med. 2005;119:187-202. 